TOC:

Synthesis of Novel Palladacycles and Their Application in the Heck and

Suzuki Reaction under Aerobic Conditions

Zhengchang Xiong, Nengdong Wang, Mingji Dai, Ang Li, Jiahua Chen*

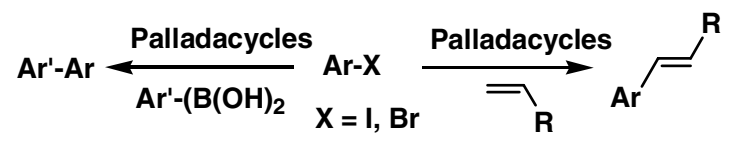
and Zhen Yang* 


\title{
Synthesis of Novel Furancarbothioamide-Based Palladacycles and Their Application in the Heck and Suzuki Reaction under Aerobic Conditions
}

\author{
Zhengchang Xiong, ${ }^{\dagger}$ Nengdong Wang, ${ }^{\dagger}$ Mingji Dai, ${ }^{\dagger}$ Ang Li, ${ }^{\dagger}$ Jiahua, Chen ${ }^{\dagger}$ \\ and Zhen Yang ${ }^{*, \dagger, \ddagger}$ \\ Key Laboratory of Bioorganic Chemistry and Molecular Engineering of Ministry of Education, College \\ of Chemistry, Beijing, 100871, China, Laboratory of Chemical Genetics, Shenzhen Graduate School of \\ Peking University, The University Town, Shenzhen, 518055, China, and VivoQuest, Inc., 711 Executive \\ Blvd, Valley Cottage, New York 10989, USA \\ zyang@chem.pku.edu.cn
}

\section{Supporting information}

\section{General Information}

All reagents were used as received and used directly without further purification. The furancarbothioamide were prepared according to the literature method (Sheibye, S.; Pedersen, B. S.; Lawesson, S.-O. Bull Soc. Chim. Belg. 1978, 87, 229.).

\section{Syntheses of Palladacycles 13-17}

$\mathrm{PdCl}_{2}(1.0 \mathrm{mmol})$ and $\mathrm{LiCl}(1.0 \mathrm{mmol})$ were dissolved in hot methanol $(15 \mathrm{~mL})$, and the generated homogeneous solution was cooled to room temperature. To this solution furancarbothioamide $(1.0 \mathrm{mmol})$ was added, and the resulted suspension was stirred overnight. The solids were filtered, washed with $\mathrm{MeOH}$, and dried in vacuum to give palladacycles 13-17 as yellow solids. 

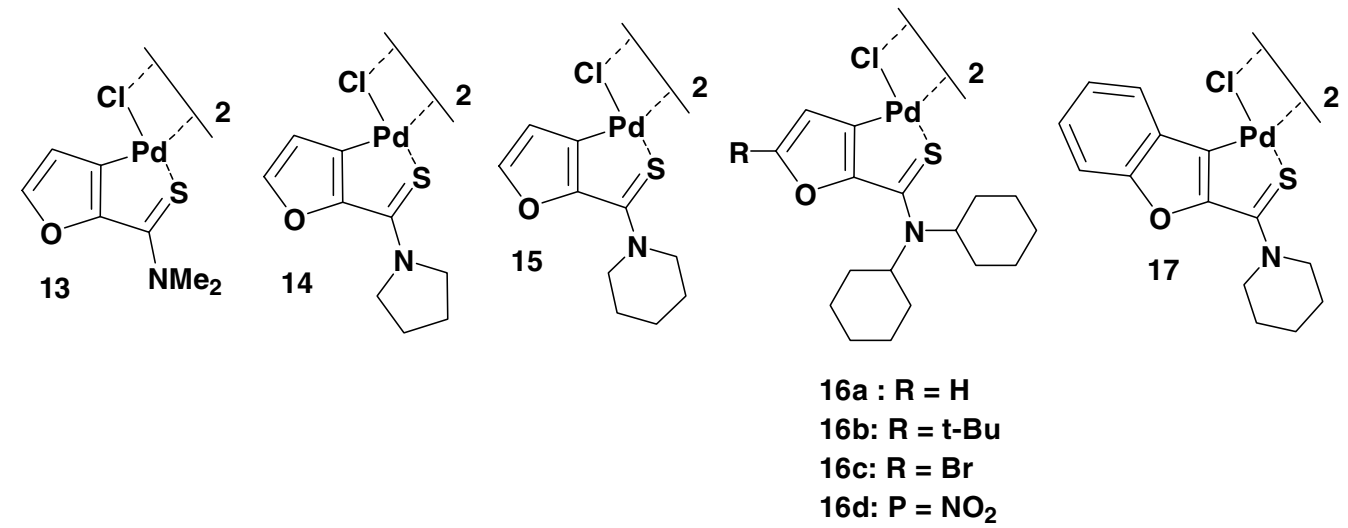

Spectral data for palladacycle 13: Yield: $72 \%$; IR $\left(\mathrm{KBr}, \mathrm{cm}^{-1}\right)$ : 1590, 1446, 1422, 1383, 1054, 981; ${ }^{1} \mathrm{H}$ NMR (300 MHz, DMSO- $\left.d_{6}\right): 83.53(\mathrm{~s}, 3 \mathrm{H}), 3.68$ (s, 3H), $7.12(\mathrm{br}, 1 \mathrm{H})$, $7.93(\mathrm{br}, 1 \mathrm{H})$.

Spectral data for palladacycle 14: Yield: $75.6 \%$; IR $\left(\mathrm{KBr}, \mathrm{cm}^{-1}\right)$ : 1581, 1447, 1387, 1059; ${ }^{1} \mathrm{H}$ NMR (300 MHz, DMSO- $\left.d_{6}\right): 1.97-2.10(\mathrm{~m}, 4 \mathrm{H}), 3.86(\mathrm{t}, J=7.0 \mathrm{~Hz}, 2 \mathrm{H})$, $4.10(\mathrm{t}, J=7.0 \mathrm{~Hz}, 2 \mathrm{H}), 7.07(\mathrm{br}, 1 \mathrm{H}), 7.90(\mathrm{br}, 1 \mathrm{H}) ;{ }^{13} \mathrm{C}$ NMR $\left(75 \mathrm{MHz}\right.$, DMSO- $\left.d_{6}\right): \bar{\delta}$ 23.44, 25.42, 52.49, 53.08, 117.1, 144.17, 145.73, 147.90, 173.85; Elemental analysis: $\left(\mathrm{C}_{9} \mathrm{H}_{10} \mathrm{ClNOPdS}\right)_{2}$ (322.12). Calcd: C, 33.56; H, 3.31; N, 4.35. Found: C, 33.52; H, $3.27 ; \mathrm{N}, 4.35$.

Spectral data for palladacycle 15: Yield: $77.3 \%$; IR $\left(\mathrm{KBr}, \mathrm{cm}^{-1}\right)$ : 2940, 1575, 1448, 1385, 1058, 974; ${ }^{1} \mathrm{H}$ NMR (300 MHz, DMSO- $\left.d_{6}\right): 1.65-1.73(\mathrm{~m}, 6 \mathrm{H}), 4.04(\mathrm{~m}, 2 \mathrm{H})$, $4.30(\mathrm{~m}, 2 \mathrm{H}), 7.03(\mathrm{br}, 1 \mathrm{H}), 7.87(\mathrm{br}, 1 \mathrm{H}) ;{ }^{13} \mathrm{C}$ NMR (75 MHz, DMSO- $\left.d_{6}\right): 023.06$, 25.84, 26.54, 51.48, 52.77, 117.53, 144.27, 145.33, 148.09, 174.21; Elemental analysis: $\left(\mathrm{C}_{10} \mathrm{H}_{12} \mathrm{ClNOPdS}\right)_{2}(336.15)$. Calcd: C, 35.73; H, 3.60; N, 4.17. Found: C, 33.76; H, $3.68 ; \mathrm{N}, 4.12$.

Spectral data for palladacycle 16a: Yield: $78.9 \%$; IR $\left(\mathrm{KBr}, \mathrm{cm}^{-1}\right)$ : 2933, 2854, 1523, $1447,1366,1057,962 ;{ }^{1} \mathrm{H}$ NMR (300 MHz, DMSO- $\left.d_{6}\right): 1.06-1.40$ (m, 8H), 1.61-1.81 (m, 12H), $3.92(\mathrm{br}, 1 \mathrm{H}), 4.95(\mathrm{br}, 1 \mathrm{H}), 7.14(\mathrm{br}, 1 \mathrm{H}), 7.90(\mathrm{br}, 1 \mathrm{H}),{ }^{13} \mathrm{C}$ NMR $(75 \mathrm{MHz}$, DMSO- $\left.d_{6}\right): \delta 24.15,24.64,25.16,29.24,29.80,61.95,65.11,117.24,145.48,146.87$, 147.90, 176.46; Elemental analysis: $\left(\mathrm{C}_{17} \mathrm{H}_{24} \mathrm{ClNOPdS}\right)_{2}$ (432.32). Calcd: C, 47.23; H, 5.60; N, 3.24. Found: C, 47.13; H, 5.55; N, 3.02 .

Spectral data for palladacycle 16b: Yield: $83.8 \%$; IR $\left(\mathrm{KBr}, \mathrm{cm}^{-1}\right)$ : 2931, 2855, 1521, 1266; ${ }^{1} \mathrm{H}$ NMR (300 MHz, DMSO- $\left.d_{6}\right): 1.05-1.50(\mathrm{~m}, 17 \mathrm{H}), 1.55-1.82(\mathrm{~m}, 12 \mathrm{H}), 3.86$ (br, 1H), $4.92(\mathrm{br}, 1 \mathrm{H}), 6.86(\mathrm{br}, 1 \mathrm{H})$; Elemental analysis: $\left(\mathrm{C}_{21} \mathrm{H}_{32} \mathrm{ClNOPdS}\right)_{2}(488.42)$. Calcd: C, 51.64; H, 6.60; N, 2.87. Found: C, 51.66; H, 6.59; N, 2.73.

Spectral data for palladacycle 16c: Yield: $86.1 \%$; IR $\left(\mathrm{KBr}, \mathrm{cm}^{-1}\right)$ : 2930, 2854, 1530, 1437, $1346 ;{ }^{1} \mathrm{H}$ NMR (300 MHz, DMSO- $\left.d_{6}\right): 1.10-1.42(\mathrm{~m}, 8 \mathrm{H}), 1.59-1.82(\mathrm{~m}, 12 \mathrm{H})$, 
3.96 (br, 1H), 4.72 (br, 1H), 7.11 (br, 1H); Elemental analysis: $\left(\mathrm{C}_{17} \mathrm{H}_{23} \mathrm{BrClNOPdS}\right)_{2}$ (511.21). Calcd: C, 39.94; H, 4.53; N, 2.74. Found: C, 40.06; H, 4.56; N, 2.59.

Spectral data for palladacycle 16d: Yield: $82.5 \%$; IR (KBr, $\left.\mathrm{cm}^{-1}\right)$ : 2934, 2857, 1524, 1442, 1344, 1080; ${ }^{1} \mathrm{H}$ NMR (300 MHz, DMSO- $\left.d_{6}\right)$ : 1.02-1.46 (m, 8H), 1.60-2.07 (m, $12 \mathrm{H}), 4.08$ (br, 1H), 4.89 (br, $1 \mathrm{H}), 7.82$ (br, $1 \mathrm{H}) ;{ }^{13} \mathrm{C}$ NMR $\left(75 \mathrm{MHz}, \mathrm{DMSO}-d_{6}\right): \delta$ 25.11, 24.60, 25.20, 28.36, 29.76, 62.07, 66.67, 115.30, 145.40, 150.03, 151.09, 178.40; Elemental analysis: $\left(\mathrm{C}_{17} \mathrm{H}_{23} \mathrm{BrClN}_{2} \mathrm{O}_{3} \mathrm{PdS}\right)_{2}$ (477.31). Calcd: C, 42.78; H, 4.86; N, 5.87. Found: C, 42.80; H, 4.91; N, 5.82.

Spectral data for palladacycle 17: Yield: $80.5 \%$; IR $\left(\mathrm{KBr}, \mathrm{cm}^{-1}\right)$ : 2930, 2854, 1510 , 1450, 1314, 1014; ${ }^{1} \mathrm{H}$ NMR (300 MHz, DMSO- $\left.d_{6}\right): 1.02-1.58(\mathrm{~m}, 8 \mathrm{H}), 1.63-2.07$ (m, $12 \mathrm{H}), 4.02$ (br, 1H), 5.11 (br, 1H), 7.21-7.26 (m, 1H), 7.45-7.49 (m, 2H), 8.70 (br, $1 \mathrm{H})$; Elemental analysis: $\left(\mathrm{C}_{21} \mathrm{H}_{26} \mathrm{BrClNOPdS}\right)_{2}$ (482.38). Calcd: C,52.29; H, 5.43; N, 2.90. Found: C, 52.56; H, 5.51; N, 2.81 .

General procedure for palladacycle 16a catalyzed Heck reaction of aryl iodides with terminal olefins

Dimethylacetamide $(2 \mathrm{~mL})$, aryliodides $(1.0 \mathrm{mmol})$, butyl acrylate $(1.2 \mathrm{mmol}) \mathrm{Et}_{3} \mathrm{~N}$ $(1.4 \mathrm{mmol})$ were added to a flask $(20 \mathrm{~mL})$. To this solution, palladacycle $16 \mathbf{a}$ in dimethylacetamide $\left(2.3 \times 10^{-3} \mathrm{M}\right.$ solution $)$ was added under aerobic conditions, and the reaction mixture was stirred at $120{ }^{\circ} \mathrm{C}$ until the complete conversion of aryliodide to product. The reaction mixture was then extracted with ethyl acetate, washed with brine and dried over $\mathrm{MgSO}_{4}$. The solvent was removed under vacuum, and the residue was purified by flash chromatography on silica gel to give the product.

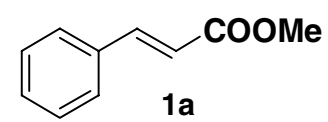

${ }^{1} \mathrm{H}$ NMR $\left(300 \mathrm{MHz}, \mathrm{CDCl}_{3}\right): 83.78(\mathrm{~s}, 3 \mathrm{H}), 6.43(\mathrm{~d}, J=15.9 \mathrm{~Hz}, 1 \mathrm{H}), 7.34-7.36(\mathrm{~m}$, $3 \mathrm{H}), 7.47-7.50(\mathrm{~m}, 2 \mathrm{H}), 7.68(\mathrm{~d}, J=16.5 \mathrm{~Hz}, 1 \mathrm{H}) ;{ }^{13} \mathrm{C} \mathrm{NMR}\left(75 \mathrm{MHz}, \mathrm{CDCl}_{3}\right): \delta 51.5$, 117.6, 127.9, 128.7, 130.1, 134.2, 144.7, 167.2; MS (EI) $\left[\mathrm{C}_{10} \mathrm{H}_{10} \mathrm{O}_{2}\right]: \mathrm{m} / \mathrm{z}\left(\mathrm{M}^{+}\right)$: Calcd 162, found 162. (Known compound, see: Crosignani, S.; White, P. D.; Linclau, B. Org. Lett. 2002, 4, 1035.)

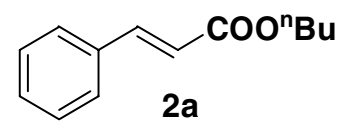

${ }^{1} \mathrm{H}$ NMR $\left(300 \mathrm{MHz}, \mathrm{CDCl}_{3}\right): \delta 0.97(\mathrm{t}, J=7.2 \mathrm{~Hz}, 3 \mathrm{H}), 1.40-1.48(\mathrm{~m}, 2 \mathrm{H}), 1.67-1.72(\mathrm{~m}$, $2 \mathrm{H}), 4.21(\mathrm{t}, \mathrm{J}=7.2 \mathrm{~Hz}, 2 \mathrm{H}), 6.42(\mathrm{~d}, J=15.9 \mathrm{~Hz}, 1 \mathrm{H}), 7.34-7.36(\mathrm{~m}, 3 \mathrm{H}), 7.47-7.50$ $(\mathrm{m}, 2 \mathrm{H}), 7.68(\mathrm{~d}, J=15.9 \mathrm{~Hz}, 1 \mathrm{H}) ;{ }^{13} \mathrm{C} \mathrm{NMR}\left(75 \mathrm{MHz}, \mathrm{CDCl}_{3}\right): 0 \overline{13} .6,19.2,30.6,64.3$, 118.2, 127.9, 128.7, 130.1, 134.3, 144.4, 167.0; MS (EI) $\left[\mathrm{C}_{13} \mathrm{H}_{16} \mathrm{O}_{2}\right]: \mathrm{m} / \mathrm{z}\left(\mathrm{M}^{+}\right):$Calcd 204, found 204. (Known compound, see: Ley S. V.; Ramario, C.; Gordon, R. S.; 
Holmes, A. B.; Morrison, A. J.; McConvey, I. F.; Shirley, I. M.; Smith, S. C.; Smith, M. D. Chem. Commun. 2002, 1134.)

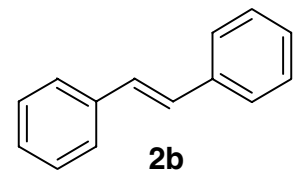

${ }^{1} \mathrm{H}$ NMR (300 MHz, $\left.\mathrm{CDCl}_{3}\right): \overline{07.10}$ (s, 2H), 7.23-7.28 (m, 2H), 7.33-7.38 (m, 4H), 7.50-7.53 (m, 4H); ${ }^{13} \mathrm{C}$ NMR (75 MHz, $\left.\mathrm{CDCl}_{3}\right)$ : 0124.7, 126.5, 127.6, 128.65, 137.3; MS (EI) $\left[\mathrm{C}_{14} \mathrm{H}_{12}\right]: \mathrm{m} / \mathrm{z}\left(\mathrm{M}^{+}\right)$: Calcd 180, found 180. (Known compound, see: Hamza, K.; Abu-Reziq, R.; Avnir, D.; Blum, J. Org. Lett. 2004, 6, 925.)

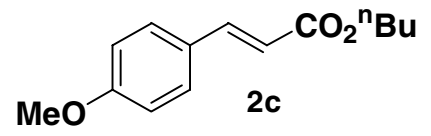

${ }^{1} \mathrm{H}$ NMR $\left(300 \mathrm{MHz}, \mathrm{CDCl}_{3}\right): \delta 0.91(\mathrm{t}, J=7.1 \mathrm{~Hz}, 3 \mathrm{H}), 1.42-1.46(\mathrm{~m}, 2 \mathrm{H}), 1.65-1.70$ $(\mathrm{m}, 2 \mathrm{H}), 3.87(\mathrm{~s}, 3 \mathrm{H}), 4.18(\mathrm{t}, J=6.6 \mathrm{~Hz}, 2 \mathrm{H}), 6.27(\mathrm{~d}, J=15.9 \mathrm{~Hz}, 1 \mathrm{H}), 6.86-6.89(\mathrm{~m}$, $2 \mathrm{H}), 7.44-7.47(\mathrm{~m}, 2 \mathrm{H}), 7.63(\mathrm{~d}, J=15.9 \mathrm{~Hz}, 1 \mathrm{H}) ;{ }^{13} \mathrm{C} \mathrm{NMR}\left(75 \mathrm{MHz}, \mathrm{CDCl}_{3}\right): \delta$ 13.93, 20.60, 30.64, 55.09, 64.03, 114.09, 115.52, 126.97, 129.49, 144.01, 161.13, 167.19; MS (EI) $\left[\mathrm{C}_{14} \mathrm{H}_{18} \mathrm{O}_{3}\right]: \mathrm{m} / \mathrm{z}\left(\mathrm{M}^{+}\right)$: Calcd 234, found 234. (Known compound, See: Bohm, V. P. W.; Hermann, W. A. Chemistry-A European Journal 2000, 6, 1017.)

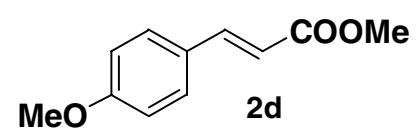

${ }^{1} \mathrm{H}$ NMR (300 MHz, $\left.\mathrm{CDCl}_{3}\right): \delta 3.75$ (s, 3H), 3.85 (s, 3H), 6.31-6.38 (d, J=10.0 Hz, 1H), 7.21-7.37 (abba, 4H), 7.94-7.99 (d, $J=10.0 \mathrm{~Hz}, 1 \mathrm{H}) ;{ }^{13} \mathrm{C} \mathrm{NMR}\left(75 \mathrm{MHz}, \mathrm{CDCl}_{3}\right): \bar{\delta}$ 51.5, 55.3, 114.3, 115.2, 127.1, 129.7, 144.5, 161.4, 167.8; MS (EI) $\left[\mathrm{C}_{11} \mathrm{H}_{12} \mathrm{O}_{3}\right]: \mathrm{m} / \mathrm{z}$ $\left(\mathrm{M}^{+}\right)$: Calcd 192, found 192. (Known compound, see: Andrus, M. B.; Song, C.; Zhang, J. Org. Lett. 2002, 4, 2079.)

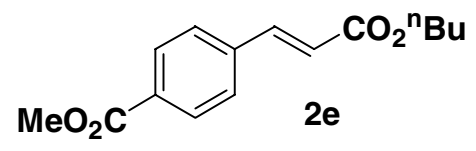

${ }^{1} \mathrm{H}$ NMR $\left(300 \mathrm{MHz}, \mathrm{CDCl}_{3}\right): \overline{0} 0.95(\mathrm{t}, J=7.2 \mathrm{~Hz}, 3 \mathrm{H}), 1.40-1.48(\mathrm{~m}, 2 \mathrm{H}), 1.67-1.72$ $(\mathrm{m}, 2 \mathrm{H}), 3.91(\mathrm{~s}, 3 \mathrm{H}), 4.22(\mathrm{t}, J=6.6 \mathrm{~Hz}), 6.54(\mathrm{~d}, J=15.9 \mathrm{~Hz}, 1 \mathrm{H}), 7.57-7.60(\mathrm{~m}, 2 \mathrm{H})$, $7.69(\mathrm{~d}, J=15.9,1 \mathrm{H}), 8.04-8.06(\mathrm{~m}, 2 \mathrm{H}) ;{ }^{13} \mathrm{C} \mathrm{NMR}\left(75 \mathrm{MHz}, \mathrm{CDCl}_{3}\right): \delta 13.6,19.1$, 30.6, 52.2, 64.5, 120.6, 127.8, 130.0, 131.2, 138.6, 143.0, 166.3, 166.5; MS (EI) $\left[\mathrm{C}_{15} \mathrm{H}_{18} \mathrm{O}_{4}\right]: \mathrm{m} / \mathrm{z}\left(\mathrm{M}^{+}\right)$: Calcd 262, found 262. (Known compound, see: Shaughnessy, K. H.; Kim, P.; Hartwig, J. F. J. Am. Chem. Soc. 1999, 121, 2123.)<smiles>CCOC(=O)C=Cc1ccc(C(C)=O)cc1</smiles> 
${ }^{1} \mathrm{H}$ NMR $\left(300 \mathrm{MHz}, \mathrm{CDCl}_{3}\right): \delta 3.82(\mathrm{~s}, 3 \mathrm{H}), 3.93$ (s, 3H), $6.52(\mathrm{~d}, J=16.2 \mathrm{~Hz}, 1 \mathrm{H})$, $7.58(\mathrm{~d}, J=9.0 \mathrm{~Hz}, 2 \mathrm{H}), 7.71(\mathrm{~d}, J=16.2 \mathrm{~Hz}, 2 \mathrm{H}), 8.05(\mathrm{~d}, J=8.1 \mathrm{~Hz}, 1 \mathrm{H}) ;{ }^{13} \mathrm{C} \mathrm{NMR}$ (75 MHz, $\left.\mathrm{CDCl}_{3}\right): \delta 51.8,52.2,120.1,127.8,130.0,131.3,138.5,143.4,166.3,166.9$; MS (EI) $\left[\mathrm{C}_{12} \mathrm{H}_{12} \mathrm{O}_{4}\right]: \mathrm{m} / \mathrm{z}\left(\mathrm{M}^{+}\right)$: Calcd 220, found 220. (Known compound, see: Villemin, D.; Jaffres, P.-A.; Nechab, B.; Courivaud, F. Tetrahedron Lett. 1997, 38, 6581.)

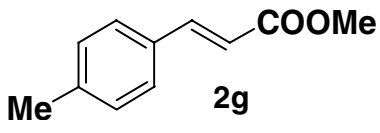

${ }^{1} \mathrm{H}$ NMR $\left(300 \mathrm{MHz}, \mathrm{CDCl}_{3}\right): \delta 2.37$ (s, 3H), 3.79 (s, 3H), 6.39(d, J=15.9 Hz, 1H), 7.18 $(\mathrm{d}, J=8.4 \mathrm{~Hz}, 2 \mathrm{H}), 7.42(\mathrm{~d}, J=8.1 \mathrm{~Hz}, 2 \mathrm{H}), 7.68(\mathrm{~d}, J=15.9 \mathrm{~Hz}, 1 \mathrm{H}) ;{ }^{13} \mathrm{C}$ NMR $(75$ $\left.\mathrm{MHz}, \mathrm{CDCl}_{3}\right): \delta 21.4,51.6,116.7,128.0,129.6,131.6,140.7,144.8,167.6$; MS (EI) $\left[\mathrm{C}_{11} \mathrm{H}_{12} \mathrm{O}_{2}\right]: \mathrm{m} / \mathrm{z}\left(\mathrm{M}^{+}\right)$: Calcd 176, found 176. (Know compound, see: Andrus, M. B.; Song, C.; Zhang, J. Org. Lett. 2002, 4, 2079.)

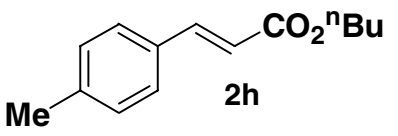

${ }^{1} \mathrm{H}$ NMR (300 MHz, $\left.\mathrm{CDCl}_{3}\right): \delta 0.95(\mathrm{t}, J=7.5 \mathrm{~Hz}, 3 \mathrm{H}), 1.41-1.46(\mathrm{~m}, 2 \mathrm{H}), 1.65-1.70$ $(\mathrm{m}, 2 \mathrm{H}), 2.34(\mathrm{~s}, 3 \mathrm{H}), 4.19(\mathrm{t}, J=6.6 \mathrm{~Hz}, 2 \mathrm{H}), 6.39(\mathrm{~d}, J=15.9 \mathrm{~Hz}, 1 \mathrm{H}), 7.16(\mathrm{~d}, J=$ $7.8 \mathrm{~Hz}, 2 \mathrm{H}), 7.40(\mathrm{~d}, J=7.8 \mathrm{~Hz}, 2 \mathrm{H}), 7.66(\mathrm{~d}, J=15.9 \mathrm{~Hz}, 1 \mathrm{H}) ;{ }^{13} \mathrm{C}$ NMR $(75 \mathrm{MHz}$, $\left.\mathrm{CDCl}_{3}\right): \delta 13.7,19.1,21.4,30.7,64.2$, 117.1, 128.0, 129.5, 131.7, 140.5, 144.5, 167.2; MS (EI) $\left[\mathrm{C}_{14} \mathrm{H}_{18} \mathrm{O}_{2}\right]: \mathrm{m} / \mathrm{z}\left(\mathrm{M}^{+}\right)$: Calcd 218, found 218. (Known compound, see: Yang, C.; Nolan, S. P. Synlett. 2001, 10, 1539.)

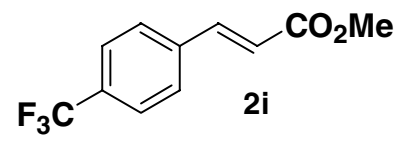

${ }^{1} \mathrm{H}$ NMR $\left(300 \mathrm{MHz}, \mathrm{CDCl}_{3}\right): \delta 3.83(\mathrm{~s}, 3 \mathrm{H}), 6.52(\mathrm{~d}, J=15.9 \mathrm{~Hz}, 1 \mathrm{H}), 7.60-7.66(\mathrm{~m}$, $4 \mathrm{H}), 7.70(\mathrm{~d}, J=15.9 \mathrm{~Hz}, 1 \mathrm{H}) ;{ }^{13} \mathrm{C} \mathrm{NMR}\left(75 \mathrm{MHz}, \mathrm{CDCl}_{3}\right): \overline{051.9}, 120.3,125.8,125.8$, 128.1, 137.7, 142.9, 166.8; MS (EI) $\left[\mathrm{C}_{11} \mathrm{H}_{9} \mathrm{~F}_{3} \mathrm{O}_{2}\right]: \mathrm{m} / \mathrm{z}\left(\mathrm{M}^{+}\right)$: Calcd 230, found 230. (Know compound, see: Speranza, G.; Di Meo, A.; Zanzola, S.; Fontana, G.; Manitto, P. Synthesis 1997, 8, 931.)

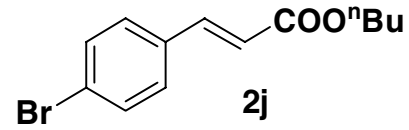

${ }^{1} \mathrm{H}$ NMR (300 MHz, $\left.\mathrm{CDCl}_{3}\right): \delta 0.99$ (t, $\left.J=7.5 \mathrm{~Hz}, 3 \mathrm{H}\right), 1.40-1.45$ (m, 2H), 1.63-1.69 $(\mathrm{m}, 2 \mathrm{H}), 4.23(\mathrm{t}, J=6.6 \mathrm{~Hz}, 2 \mathrm{H}), 6.35(\mathrm{~d}, J=15.9 \mathrm{~Hz}, 1 \mathrm{H}), 7.41(\mathrm{~d}, J=3.0 \mathrm{~Hz}, 2 \mathrm{H})$, $7.51(\mathrm{~d}, J=3.0 \mathrm{~Hz}, 2 \mathrm{H}), 7.63(\mathrm{~d}, J=15.9 \mathrm{~Hz}, 1 \mathrm{H}) ;{ }^{13} \mathrm{C} \mathrm{NMR}\left(75 \mathrm{MHz}, \mathrm{CDCl}_{3}\right): \delta 13.7$, $19.1,30.7,64.5,118.9,124.4,129.4,132.0,133.3,143.1,166.7 ; \mathrm{MS} \mathrm{m} / \mathrm{z}(\mathrm{EI})$ $\left[\mathrm{C}_{13} \mathrm{H}_{15} \mathrm{BrO}_{2}\right]: \mathrm{m} / \mathrm{z}\left(\mathrm{M}^{+}\right)$: Calcd $284\left(\mathrm{M}^{+},{ }^{81} \mathrm{Br}\right), 282\left(\mathrm{M}^{+},{ }^{79} \mathrm{Br}\right)$, found 282. (Know compound, see: Park, S. B.; Alper, H. Org. Lett. 2003, 5, 3209.) 


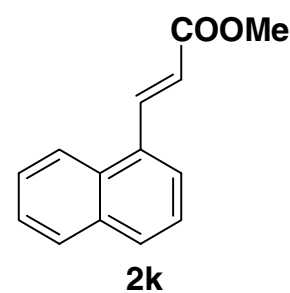

${ }^{1} \mathrm{H}$ NMR $\left(300 \mathrm{MHz}, \mathrm{CDCl}_{3}\right): \delta 3.72(\mathrm{~s}, 3 \mathrm{H}), 6.38$ (d, $\left.J=15.9 \mathrm{~Hz}, 1 \mathrm{H}\right), 7.31-7.44(\mathrm{~m}$, $3 \mathrm{H}), 7.58(\mathrm{~d}, J=7.2 \mathrm{~Hz}, 1 \mathrm{H}), 7.69-7.74(\mathrm{~m}, 2 \mathrm{H}), 8.05(\mathrm{~d}, J=7.2 \mathrm{~Hz}, 1 \mathrm{H}), 8.43(\mathrm{~d}, J=$ $15.9 \mathrm{~Hz}, 1 \mathrm{H}) ;{ }^{13} \mathrm{C}$ NMR $\left(75 \mathrm{MHz}, \mathrm{CDCl}_{3}\right): \delta 51.6,120.3,123.2,124.9,125.3,126.1$, 126.7, 128.6, 130.4, 131.3, 131.5, 133.5, 141.7, 167.2; MS (EI) $\left[\mathrm{C}_{14} \mathrm{H}_{12} \mathrm{O}_{2}\right]: \mathrm{m} / \mathrm{z}\left(\mathrm{M}^{+}\right)$: Calcd 212, found 212. (Know compound, see Andrus, M. B.; Song, C.; Zhang, J. Org. Lett. 2002, 4, 2079.)

\section{Typical experiment for the Heck reaction of aryl bromides and olefins}

Dimethylacetamide $(2 \mathrm{~mL})$, arylbromides $(1.0 \mathrm{mmol})$, butyl acrylate $(1.2 \mathrm{mmol})$, $\mathrm{NaOAc}(1.2 \mathrm{mmol}), \mathrm{n}-\mathrm{BuNBr}(0.2 \mathrm{mmol})$ were added to a flask $(20 \mathrm{~mL})$. To this solution was added palladacycle 16a in dimethylacetamide $\left(2.3 \times 10^{-3} \mathrm{M}\right.$ solution) under aerobic conditions, and the reaction mixture was stirred at $120{ }^{\circ} \mathrm{C}$ until the complete conversion of aryl bromide to the product. The reaction mixture was then extracted with ethyl acetate, washed with brine and dried over $\mathrm{MgSO}_{4}$. The solvent was removed under vacuum; the residue was purified by flash chromatography on silica gel to give the product.

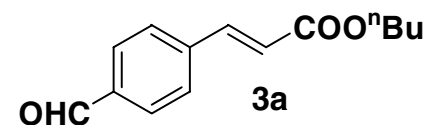

${ }^{1} \mathrm{H}$ NMR (300 MHz, $\left.\mathrm{CDCl}_{3}\right): \delta 0.97(\mathrm{t}, J=7.5 \mathrm{~Hz}, 3 \mathrm{H}), 1.40-1.45(\mathrm{~m}, 2 \mathrm{H}), 1.65-1.70$ $(\mathrm{m}, 2 \mathrm{H}), 4.23(\mathrm{t}, J=7.5 \mathrm{~Hz}, 2 \mathrm{H}), 6.55(\mathrm{~d}, J=16.2 \mathrm{~Hz}, 1 \mathrm{H}), 7.66-7.69(\mathrm{~m}, 3 \mathrm{H}), 7.90(\mathrm{~d}$, $J=8.1 \mathrm{~Hz}, 2 \mathrm{H}), 10.01(\mathrm{~s}, 1 \mathrm{H}) ;{ }^{13} \mathrm{C} \mathrm{NMR}\left(75 \mathrm{MHz}, \mathrm{CDCl}_{3}\right): \delta 13.6,19.0,30.6,64.5$, 121.3, 128.4, 130.0, 137.0, 139.9, 142.6, 166.2, 191.3; MS (EI) $\left[\mathrm{C}_{9} \mathrm{H}_{7} \mathrm{O}\right]: \mathrm{m} / \mathrm{z}\left(\mathrm{M}^{+}\right)$: Calcd 232, found 232. (Known compound, see: Yao, Q.; Kinney, E. P.; Yang, Z. J. Org. Chem. 2003, 68, 7528.).

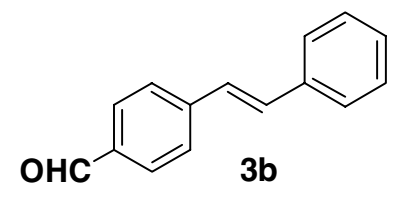

${ }^{1} \mathrm{H}$ NMR (300 MHz, $\left.\mathrm{CDCl}_{3}\right)$ : $77.08-7.40(\mathrm{~m}, 5 \mathrm{H}), 7.52(\mathrm{~d}, J=7.2 \mathrm{~Hz}, 2 \mathrm{H}), 7.63(\mathrm{~d}, J=$ $7.8 \mathrm{~Hz}, 2 \mathrm{H}), 7.85(\mathrm{~d}, J=7.8 \mathrm{~Hz}, 2 \mathrm{H}), 9.97(\mathrm{~s}, 1 \mathrm{H}) ;{ }^{13} \mathrm{C} \mathrm{NMR}\left(75 \mathrm{MHz}, \mathrm{CDCl}_{3}\right): \delta 126.8$, 127.2, 128.4, 128.8, 130.2, 132.1, 135.2, 136.4, 143.3, 191.6; MS (EI) $\left[\mathrm{C}_{15} \mathrm{H}_{12} \mathrm{O}\right]: \mathrm{m} / \mathrm{z}$ $\left(\mathrm{M}^{+}\right)$: Calcd 208, found 208. (Known compound, see: Yao, Q.; Kinney, E. P.; Yang, Z. J. Org. Chem. 2003, 68, 7528.) 


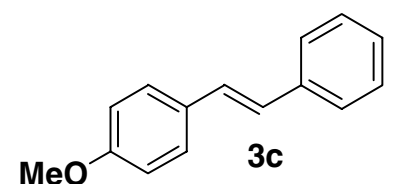

${ }^{1} \mathrm{H}$ NMR (300 MHz, $\left.\mathrm{CDCl}_{3}\right): \delta 3.90$ (s, 3H), 6.96-7.31 (m, 4H), 7.32-7.45 (m, 3H), 7.52-7.58 (m, 4H); ${ }^{13} \mathrm{C}$ NMR $\left(75 \mathrm{MHz}, \mathrm{CDCl}_{3}\right): \delta 55.3,114.1,126.2,126.5,127.2$, 127.7, 128.1, 128.6, 130.1, 137.6, 159.2; MS (EI) $\left[\mathrm{C}_{15} \mathrm{H}_{14} \mathrm{O}\right]: \mathrm{m} / \mathrm{z}\left(\mathrm{M}^{+}\right):$Calcd 210, found 210. (Known compound, see: Yang, D.; Chen, Y.-C.; Zhu, N.-Y. Org. Lett. 2004, 6, 1577.)<smiles>CCOC(=O)/C=C/c1ccc([N+](=O)[O-])cc1</smiles>

${ }^{1} \mathrm{H}$ NMR $\left(300 \mathrm{MHz}, \mathrm{CDCl}_{3}\right): \delta 0.97(\mathrm{t}, J=7.2 \mathrm{~Hz}, 3 \mathrm{H}), 1.41-1.48(\mathrm{~m}, 2 \mathrm{H}), 1.68-1.73$ $(\mathrm{m}, 2 \mathrm{H}), 4.24(\mathrm{t}, J=7.2 \mathrm{~Hz}, 2 \mathrm{H}), 6.58(\mathrm{~d}, J=15.9 \mathrm{~Hz}, 1 \mathrm{H}), 7.66-7.73(\mathrm{~m}, 3 \mathrm{H}), 8.25(\mathrm{~d}$, $J=8.7 \mathrm{~Hz}, 2 \mathrm{H}) ;{ }^{13} \mathrm{C}$ NMR $\left(75 \mathrm{MHz}, \mathrm{CDCl}_{3}\right): \delta 13.6,19.0,30.6,64.8,122.5,124.0$, 128.5, 140.5, 141.5, 148.3, 166.0; MS (EI) $\left[\mathrm{C}_{13} \mathrm{H}_{15} \mathrm{NO}_{4}\right]: \mathrm{m} / \mathrm{z}\left(\mathrm{M}^{+}\right)$: Calcd 249, found 249. (Known compound, see: de Vries, A. H. M.; Mulders, J. M. C. A.; Mommers, J. H. M.; Henderickx, H. J. W.; de Vries, J. G. Org. Lett. 2003, 5, 3285.)

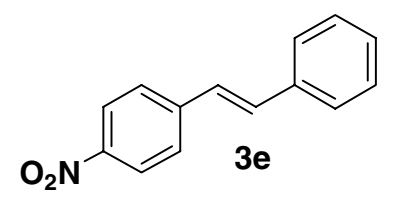

${ }^{1} \mathrm{H}$ NMR $\left(300 \mathrm{MHz}, \mathrm{CDCl}_{3}\right): \overline{0} 7.10-7.42(\mathrm{~m}, 5 \mathrm{H}), 7.54(\mathrm{~d}, J=7.2 \mathrm{~Hz}, 2 \mathrm{H}), 7.65(\mathrm{~d}, J=$ $7.8 \mathrm{~Hz}, 2 \mathrm{H}), 8.20(\mathrm{~d}, J=8.4 \mathrm{~Hz}, 2 \mathrm{H}) ;{ }^{13} \mathrm{C} \mathrm{NMR}\left(75 \mathrm{MHz}, \mathrm{CDCl}_{3}\right): 0124.1,126.2$, 126.8, 127.0, 128.8, 133.2, 136.1, 143.8, 146.7; MS (EI) $\left[\mathrm{C}_{14} \mathrm{H}_{11} \mathrm{NO}_{2}\right]: \mathrm{m} / \mathrm{z}\left(\mathrm{M}^{+}\right)$: Calcd 225, found 225. (Known compound, see: Masllorens, J.; Moreno-Manas, M.; Pla-Quintana, A.; Roglans, A. Org. Lett. 2003, 5, 1559.)

\section{General procedure for the Suzuki reaction of Aryl halides with boronic acids}

Aryliodides or bromides (1.0 mmol), arylboronic acid (1.2 mmol), $\mathrm{K}_{2} \mathrm{CO}_{3}(2.0$ $\mathrm{mol})$, palladacycles 16a in dimethylacetamide $\left(2.3 \times 10^{-3} \mathrm{M}\right.$ solution) and a solution of dimethylacetamide $/ \mathrm{H}_{2} \mathrm{O}(1.5 \mathrm{ml} / 0.5 \mathrm{ml})$ were added to a flask $(20 \mathrm{~mL})$ under aerobic conditions, and the reaction mixture was stirred at $100 \square$ until complete conversion of aryl halide to the product. The reaction mixture was extracted with ethyl acetate, washed with brine, and dried over $\mathrm{MgSO}_{4}$. The solvent was removed under vacuum and the residue was purified by a flash chromatography on silica gel to give the product.

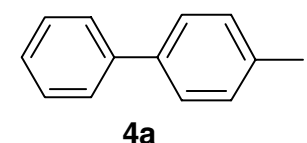


${ }^{1} \mathrm{H}$ NMR $\left(300 \mathrm{MHz}, \mathrm{CDCl}_{3}\right): \bar{\delta} 3.79$ (s, 3H), 7.30-7.36 (m, 2H), 7.40-7.45 (m, 4H), 7.57-7.60(m, 4H); ${ }^{13} \mathrm{C}$ NMR $\left(75 \mathrm{MHz}, \mathrm{CDCl}_{3}\right): 0127.1,127.2,128.7,141.2 ; \mathrm{MS}$ (EI) $\left[\mathrm{C}_{12} \mathrm{H}_{10}\right]: \mathrm{m} / \mathrm{z}\left(\mathrm{M}^{+}\right)$: Calcd 154, found 154. (Known compound, see: Su, W.; Urgaonkar, S.; Verkade, J. G. Org. Lett. 2004, 6, 1421.

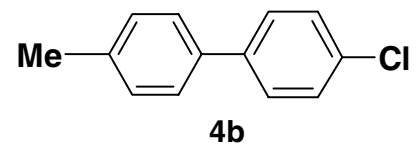

${ }^{1} \mathrm{H}$ NMR (300 MHz, $\left.\mathrm{CDCl}_{3}\right): \bar{\delta} 2.38$ (s, 3H), 7.21-7.24 (m, 2H), 7.35-7.49 (m, 6H); ${ }^{13} \mathrm{C}$ NMR (75 MHz, $\left.\mathrm{CDCl}_{3}\right): \delta 21.0,126.7,128.1,128.8,129.5,132.9,137.0,137.4,139.5$; MS (EI) $\left[\mathrm{C}_{13} \mathrm{H}_{11} \mathrm{Cl}\right]: \mathrm{m} / \mathrm{z}\left(\mathrm{M}^{+}\right)$: Calcd $204\left(\mathrm{M}^{+}, \mathrm{Cl}^{37}\right), 202\left(\mathrm{M}^{+}, \mathrm{Cl}^{35}\right)$, found 202, 204. (Know compound, see: Dubbaka, S. R.; Vogel, P. Org. Lett. 2004, 6, 95.)

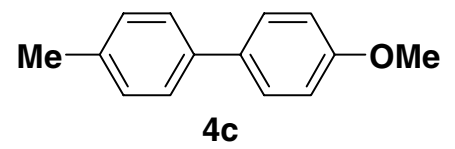

${ }^{1} \mathrm{H}$ NMR (300 MHz, $\left.\mathrm{CDCl}_{3}\right): \delta 2.37$ (s, 3H), 3.82 (s, 3H), $6.93(\mathrm{~d}, J=8.7 \mathrm{~Hz}, 2 \mathrm{H})$, 7.20-7.22 (m, 2H), 7.42-7.51 (m, 4H); $\left.{ }^{13} \mathrm{C} \mathrm{NMR} \mathrm{(75} \mathrm{MHz,} \mathrm{CDCl}_{3}\right)$ : 21.0, 55.3, 114.1, 126.5, 127.9, 129.4, 133.7, 136.3, 137.9, 158.9; MS (EI) $\left[\mathrm{C}_{14} \mathrm{H}_{14} \mathrm{O}\right]: \mathrm{m} / \mathrm{z}\left(\mathrm{M}^{+}\right):$Calcd 198, found 198. (Known compound, see: Percec, V.; Golding, G. M.; Smidrkal, J.; Weichold, O. J. Org. Chem. 2004, 69, 3447.)

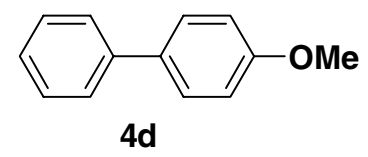

${ }^{1} \mathrm{H}$ NMR $\left(300 \mathrm{MHz}, \mathrm{CDCl}_{3}\right): \delta 3.80(\mathrm{~s}, 3 \mathrm{H}), 6.98(\mathrm{~d}, J=8.7 \mathrm{~Hz}, 2 \mathrm{H}), 7.27-7.32(\mathrm{~m}, 1 \mathrm{H})$, 7.38-7.43 (m, 2H), 7.51-7.60 (m, 4H); $\left.{ }^{13} \mathrm{C} \mathrm{NMR} \mathrm{(75} \mathrm{MHz,} \mathrm{CDCl}_{3}\right)$ : $855.3,114.2,126.6$, 126.7, 128.1, 128.7, 133.8, 140.8, 159.1; MS (EI) $\left[\mathrm{C}_{13} \mathrm{H}_{12} \mathrm{O}\right]: \mathrm{m} / \mathrm{z}\left(\mathrm{M}^{+}\right)$: Calcd 184, found 184. (Known compound, see: Okamoto, K.; Akiyama, R.; Kobayashi, S. Org. Lett. 2004, 6, 1987.)

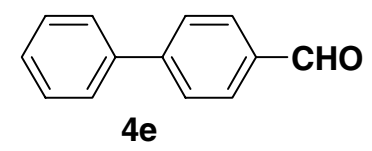

${ }^{1} \mathrm{H}$ NMR (300 MHz, $\left.\mathrm{CDCl}_{3}\right)$ :ठ̄7.42-7.51 (m, 3H), 7.62-7.66 (m, 2H), 7.74-7.77 (m, 2H), 7.94-7.97 (m, 2H), $10.06(\mathrm{~s}, 1 \mathrm{H}) ;{ }^{13} \mathrm{C} \mathrm{NMR}\left(75 \mathrm{MHz}, \mathrm{CDCl}_{3}\right): 0127.3,127.6$, 128.4, 129.0, 130.2, 135.2, 139.7, 147.2, 191.9; MS (EI) $\left[\mathrm{C}_{13} \mathrm{H}_{10} \mathrm{O}\right]: \mathrm{m} / \mathrm{z}\left(\mathrm{M}^{+}\right)$: Calcd 182, found 182. (Known compound, see: Mutule, I.; Suna, E. Tetrahedron Lett. 2004, 45,3909 .)

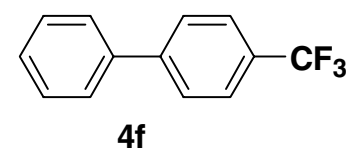


${ }^{1} \mathrm{H}$ NMR (300 MHz, $\left.\mathrm{CDCl}_{3}\right): \overline{0} 7.38-7.48(\mathrm{~m}, 3 \mathrm{H}), 7.50-7.56(\mathrm{~m}, 2 \mathrm{H}), 7.60(\mathrm{~m}, 4 \mathrm{H}) ;{ }^{13} \mathrm{C}$ NMR (75 MHz, $\left.\mathrm{CDCl}_{3}\right): \delta 125.6,126.1,127.3,127.4,128.2,128.7,129.0,139.7,144.7$; MS (EI) $\left[\mathrm{C}_{13} \mathrm{H}_{9} \mathrm{~F}_{3}\right]: \mathrm{m} / \mathrm{z}\left(\mathrm{M}^{+}\right)$: Calcd 222, found 222. (Known compound, see: Navarro, O.; Kaur, H.; Mahjoor, P.; Nolan, S. P. J. Org. Chem. 2004, 69, 3173.)

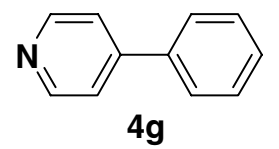

${ }^{1} \mathrm{H}$ NMR (300 MHz, $\left.\mathrm{CDCl}_{3}\right): \bar{\delta} 7.48-7.54(\mathrm{~m}, 5 \mathrm{H}), 7.65-7.67$ (d, J = $\left.6 \mathrm{~Hz}, 2 \mathrm{H}\right), 8.69$ (d, $\mathrm{J}=6 \mathrm{~Hz}, 2 \mathrm{H}) ;{ }^{13} \mathrm{C} \mathrm{NMR}\left(75 \mathrm{MHz}, \mathrm{CDCl}_{3}\right): \delta 121.6,126.9,129.0,129.0,138.0,148.3$, 150.1; MS (EI) $\left[\mathrm{C}_{11} \mathrm{H}_{9} \mathrm{~N}\right]: \mathrm{m} / \mathrm{z}\left(\mathrm{M}^{+}\right)$: Calcd 155, found 155. (Kn compound, see: Feuerstein, M.; Doucet, H.; Santelli, M. J. Organomet. Chem. 2003, 687, 327.)

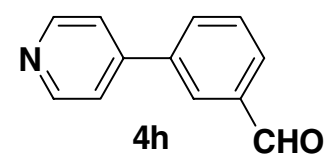

${ }^{1} \mathrm{H}$ NMR $\left(300 \mathrm{MHz}, \mathrm{CDCl}_{3}\right)$ : $\mathbf{0 7 . 5 4 - 7 . 5 6}(\mathrm{dd}, J=2.0,4.8 \mathrm{~Hz}, 2 \mathrm{H}), 7.68(\mathrm{t}, J=7.8 \mathrm{~Hz}, 1$ H), 7.89-7.97 (m, 2H) , 8.15 (s, 1H), 8.70-8.72 (dd, $J=2.0,4.8 \mathrm{~Hz}, 2 \mathrm{H}), 10.1$ (s 1H); ${ }^{13} \mathrm{C}$ NMR (75 MHz, $\left.\mathrm{CDCl}_{3}\right): \delta 121.5,127.7,129.8,130.3,132.7,137.0,139.0,146.8$, 150.3, 191.7; $\mathrm{MS}(\mathrm{EI})\left[\mathrm{C}_{12} \mathrm{H}_{9} \mathrm{NO}\right]: \mathrm{m} / \mathrm{z}\left(\mathrm{M}^{+}\right)$: Calcd 183, found 183. (Known compound, see: Massa, M. A.; Spangler, D. P.; Durley, R. C.; Hickory, B. S.; Connolly, D. T.; Witherbee, B. J.; Smith, M. E.; Sikorski, J. A. Bioorg \& Med. Chem. Lett. 2001, $11,1625$.

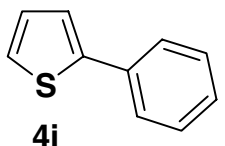

${ }^{1} \mathrm{H}$ NMR (300 MHz, $\left.\mathrm{CDCl}_{3}\right): \overline{0} 7.11-7.14(\mathrm{~m}, 1 \mathrm{H})$, 7.30-7.45 (m, 5H), 7.66-7.69 (m, $2 \mathrm{H}) ;{ }^{13} \mathrm{C}$ NMR $\left(75 \mathrm{MHz}, \mathrm{CDCl}_{3}\right): \delta 123.0,124.7,125.9,127.4,128.0,128.8,134.4$, 144.4; MS (EI) $\left[\mathrm{C}_{10} \mathrm{H}_{8} \mathrm{~S}\right]: \mathrm{m} / \mathrm{z}\left(\mathrm{M}^{+}\right)$: Calcd 160, found 160. (Known compound, see: Wolf, C.; Lerebours, R. Org. Lett. 2004, 6, 1147.)<smiles>c1ccc(-c2cccc3ccccc23)cc1</smiles>

$4 \mathbf{j}$

${ }^{1} \mathrm{H}$ NMR (300 MHz, CDCl $): \delta 7.56(\mathrm{~m}, 9 \mathrm{H}), 7.93(\mathrm{~m}, 1 \mathrm{H}), 7.96(\mathrm{~m}, 2 \mathrm{H}) ;{ }^{13} \mathrm{C}$ NMR $(75$ $\left.\mathrm{MHz}, \mathrm{CDCl}_{3}\right): \delta 125.6,125.8,125.9$, 126.3, 127.3, 127.4, 127.6, 128.2, 128.4, 130.3, 132.6, 133.6, 140.6, 141.1; MS (EI) $\left[\mathrm{C}_{16} \mathrm{H}_{12}\right]: \mathrm{m} / \mathrm{z}\left(\mathrm{M}^{+}\right)$: Calcd 204, found 204. (Known compound, see: Percec, V.; Golding, G. M.; Smidrkal, J.; Weichold, O. J. Org. Chem. 2004, 69, 3447.) 


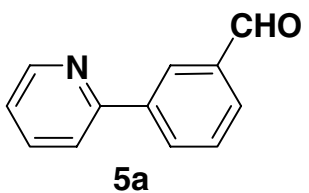

${ }^{1} \mathrm{H}$ NMR $\left(300 \mathrm{MHz}, \mathrm{CDCl}_{3}\right): \delta 7.39-7.44(\mathrm{~m}, 1 \mathrm{H}), 7.67(\mathrm{t}, J=7.5 \mathrm{~Hz}, 1 \mathrm{H}), 7.84-7.63$ $(\mathrm{m}, 1 \mathrm{H}), 7.85-7.95(\mathrm{~m}, 3 \mathrm{H}), 8.09-8.11(\mathrm{~m}, 1 \mathrm{H}), 8.90(\mathrm{~s}, 1 \mathrm{H}), 10.11(\mathrm{~s}, 1 \mathrm{H}) ;{ }^{13} \mathrm{C} \mathrm{NMR}$ (75 MHz, $\left.\mathrm{CDCl}_{3}\right): \delta 123.6,127.7,129.4,129.7,132.7,134.2,135.1,136.9,138.6$, 148.0, 149.0, 191.8; MS (EI) $\left[\mathrm{C}_{12} \mathrm{H}_{9} \mathrm{NO}\right]: \mathrm{m} / \mathrm{z}\left(\mathrm{M}^{+}\right)$: Calcd 183, found 183. (Known compound, see: Massa, M. A.; Spangler, D. P.; Durley, R. C.; Hickory, B. S.; Connolly, D. T.; Witherbee, B. J.; Smith, M. E.; Sikorski, J. A. Bioorga. \& Med. Chem. Lett. 2001, 11, 1625.)

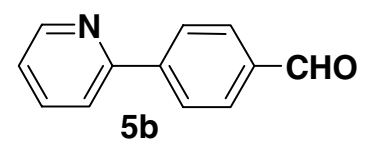

${ }^{1} \mathrm{H}$ NMR (300 MHz, $\left.\mathrm{CDCl}_{3}\right)$ :07.27-7.30 (m, 1H), $7.78(\mathrm{t}, J=7.5 \mathrm{~Hz}, 2 \mathrm{H}), 7.97(\mathrm{~m}$, 2H), $8.15(\mathrm{~m}, 2 \mathrm{H}), 8.72(\mathrm{~m}, 1 \mathrm{H}), 10.06(\mathrm{~s}, 1 \mathrm{H}) ;{ }^{13} \mathrm{C} \mathrm{NMR}\left(75 \mathrm{MHz}, \mathrm{CDCl}_{3}\right): \delta 191.6$, 155.6, 149.7, 144.7, 136.8, 136.2, 128.9, 128.8, 122.3, 121.6; MS (EI) $\left[\mathrm{C}_{12} \mathrm{H}_{9} \mathrm{NO}\right]: \mathrm{m} / \mathrm{z}$ $\left(\mathrm{M}^{+}\right)$: Calcd 183, found 183. (Known compound, see: Xu, Z.; Singh, J.; Schwinden, M. D.; Zheng, B.; Kissick, T. P.; Patel, B.; Humora, M. J.; Quiroz, F.; Dong, L.; Hsieh, D.; Heikes, J. E.; Pudipeddi, M.; Lindrud, M. D.; Srivastava, S. K.; Kronenthal, D. R.; Mueller, R. H. Organic Process Research \& Development 2002, 6, 323.)

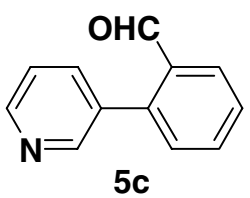

${ }^{1} \mathrm{H}$ NMR (300 MHz, $\left.\mathrm{CDCl}_{3}\right): \overline{0} 7.42-7.46(\mathrm{~m}, 2 \mathrm{H}), 7.58(\mathrm{t}, J=7.8 \mathrm{~Hz}, 1 \mathrm{H}), 7.68-7.75$ $(\mathrm{m}, 2 \mathrm{H}), 7.8 .05-8.09(\mathrm{~m}, 1 \mathrm{H}), 8.67-8.72(\mathrm{~m}, 2 \mathrm{H}), 9.99(\mathrm{~s}, 1 \mathrm{H}) ;{ }^{13} \mathrm{C}$ NMR $(75 \mathrm{MHz}$, $\left.\mathrm{CDCl}_{3}\right): \delta 123.1,128.4,128.6,130.9,133.6,133.7,133.8,137.1,141.7,149.2,150.0$, 191.2; MS (EI) $\left[\mathrm{C}_{12} \mathrm{H}_{9} \mathrm{NO}\right]: \mathrm{m} / \mathrm{z}\left(\mathrm{M}^{+}\right)$: Calcd 183, found 183. (Known compound, see: O'Shea, D. F.; Sharp, J. T. J. Chem. Soc., Perkin Trans. Org. and Bio-Org. Chem. 1996, $6,515$.

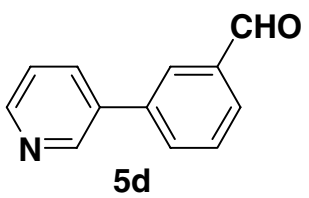

${ }^{1} \mathrm{H} \mathrm{NMR}\left(300 \mathrm{MHz}, \mathrm{CDCl}_{3}\right): \overline{0} 7.40(\mathrm{~m}, 1 \mathrm{H}), 7.64(\mathrm{~m}, 1 \mathrm{H}), 7.87(\mathrm{~m}, 1 \mathrm{H}), 7.92(\mathrm{~m}, 2 \mathrm{H})$, $8.12(\mathrm{~m}, 1 \mathrm{H}), 8.62(\mathrm{~m}, 1 \mathrm{H}), 8.83(\mathrm{~m}, 1 \mathrm{H}), 10.11(\mathrm{~s}, 1 \mathrm{H}) ;{ }^{13} \mathrm{C} \mathrm{NMR}\left(75 \mathrm{MHz}, \mathrm{CDCl}_{3}\right)$ : ס123.6, 127.6, 128.3 128,6, 132.7, 136.5, 146.0, 148.2, 191.7; MS (EI) [C $\left.{ }_{12} \mathrm{H}_{9} \mathrm{NO}\right]$ : $\mathrm{m} / \mathrm{z}\left(\mathrm{M}^{+}\right)$: Calcd 183, found 183. (Known compound, see: Hashizume, H.; Ito, H.; Kanaya, N.; Nagashima, H.; Usui, H.; Oshima, R.; Kanao, M.; Tomoda, H.; Sunazuka, T. Heterocycle 1994, 38, 1551.) 


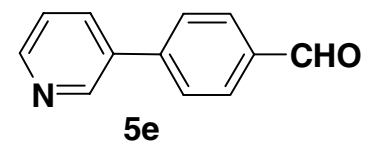

${ }^{1} \mathrm{H}$ NMR (300 MHz, $\left.\mathrm{CDCl}_{3}\right): \delta 7.41-7.45(\mathrm{~m}, 1 \mathrm{H}), 7.76$ (d, $\left.J=8.4 \mathrm{~Hz}, 2 \mathrm{H}\right), 7.92-8.01$ $(\mathrm{m}, 3 \mathrm{H}), 8.66-8.68(\mathrm{~m}, 1 \mathrm{H}), 8.91(\mathrm{~d}, J=2.1 \mathrm{~Hz}, 1 \mathrm{H}), 10.08(\mathrm{~s}, 1 \mathrm{H}) ;{ }^{13} \mathrm{C} \mathrm{NMR}(75 \mathrm{MHz}$, $\left.\mathrm{CDCl}_{3}\right): \delta 123.5,127.2,130.2,134.4,135.0,135.5,143.4,148.0,149.2,191.5 ; \mathrm{MS}$ (EI) $\left[\mathrm{C}_{12} \mathrm{H}_{9} \mathrm{NO}\right]: \mathrm{m} / \mathrm{z}\left(\mathrm{M}^{+}\right)$: Calcd 183, found 183. (Known compound, see: Cioffi, C. L.; Spencer, W. T.; Richards, J. J.; Herr, R. J. J. Org. Chem. 2004, 69, 2210.)

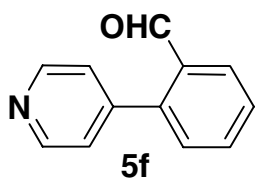

${ }^{1} \mathrm{H}$ NMR $\left(300 \mathrm{MHz}, \mathrm{CDCl}_{3}\right): \delta 7.33-7.74(\mathrm{~m}, 5 \mathrm{H}), 8.04-8.07(\mathrm{~m}, 1 \mathrm{H}), 8.65(\mathrm{~d}, J=6.0$ $\mathrm{Hz}, 2 \mathrm{H}), 9.99$ (s, 1H); ${ }^{13} \mathrm{C} \mathrm{NMR}\left(75 \mathrm{MHz}, \mathrm{CDCl}_{3}\right): \delta 124.5,128.1,128.8,130.1,133.2$, 133.7, 142.4, 145.6, 149.6, 190.9; MS (EI) $\left[\mathrm{C}_{12} \mathrm{H}_{9} \mathrm{NO}\right]: \mathrm{m} / \mathrm{z}\left(\mathrm{M}^{+}\right)$: Calcd 183, found 183. (Known compound, see: O'Shea, D. F.; Sharp, J. T. J. Chem. Soc., Perkin Trans. Org. and Bio-Org. Chem. 1996, 6, 515.)

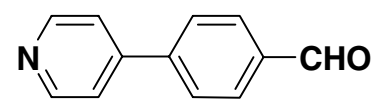

$5 g$

${ }^{1} \mathrm{H} \mathrm{NMR}\left(300 \mathrm{MHz}, \mathrm{CDCl}_{3}\right): 07.57(\mathrm{dd}, J=1.5,4.8 \mathrm{~Hz}, 2 \mathrm{H}), 7.82(\mathrm{dd}, J=1.8,7.8 \mathrm{~Hz}$, $2 \mathrm{H}), 8.01(\mathrm{dd}, J=1.8,6.6 \mathrm{~Hz}, 2 \mathrm{H}), 8.75 \quad(\mathrm{dd}, J=1.8,4.8 \mathrm{~Hz}, 2 \mathrm{H}), 10.09(\mathrm{~s} 1 \mathrm{H}) ;{ }^{13} \mathrm{C}$ NMR (75 MHz, $\left.\mathrm{CDCl}_{3}\right)$ :ס121.6, 127.4, 130.1, 136.2, 143.3, 146.8, 149.7, 191.3; MS (EI) $\left[\mathrm{C}_{12} \mathrm{H}_{9} \mathrm{NO}\right]: \mathrm{m} / \mathrm{z}\left(\mathrm{M}^{+}\right)$: Calcd 183, found 183. (Known compound, see: Nicolaou, $\mathrm{K}$. C.; Baran, P. S.; Zhong, Y-L. J. Am. Chem. Soc. 2001, 123, 3183.) 